\title{
Estado nutricional e anemia ferropriva em gestantes: relação com o peso da criança ao nascer
}

\author{
Nutritional status and iron-deficiency anemia in pregnant \\ women: relationship with the weight of the child at birth
}

\author{
Daniela da Silva ROCHA ${ }^{1}$ \\ Michele Pereira NETTO \\ Sílvia Eloiza PRIORE ${ }^{3}$ \\ Nerilda Martins Miranda de LIMA ${ }^{4}$ \\ Lina Enriqueta Frandsen Paez de Lima ROSADO ${ }^{3}$ \\ Sylvia do Carmo Castro FRANCESCHINI ${ }^{3}$
}

\section{RES U M O}

\section{Objetivo}

Avaliar o estado nutricional e a prevalência de anemia durante a gestação e correlacioná-los com o peso do recém-nascido.

\section{Métodos}

Foi realizado estudo transversal com gestantes que realizaram o pré-natal no único serviço público de saúde do município de Viçosa, MG, no período de dezembro de 2002 a maio de 2003. Foi aplicado questionário com informações maternas e realizada dosagem de hemoglobina através do Hemocue, além da obtenção de dados de peso e estatura da gestante. As informações referentes aos recém-nascidos foram obtidas no programa Sistema de Informação de Nascidos Vivos/MS.

\section{Resultados}

Foram avaliadas 168 gestantes de baixo nível socioeconômico e baixa escolaridade. Encontraram-se 41,3\% de gestantes com estado nutricional pré-gestacional inadequado, sendo $25,7 \%$ com baixo peso e $17,4 \%$ com sobrepeso ou obesidade. A maioria das gestantes apresentou ganho de peso inadequado durante a gestação. A prevalência total de anemia ferropriva foi de $21,4 \%$, sendo que essa aumentou com a idade gestacional. A freqüência de baixo peso e peso insuficiente ao nascer foi de 8,9\% e 28,6\%, respectivamente. As variáveis antropométricas (peso pré-gestacional, estatura, índice de massa corporal pré-gestacional e ganho de peso total) apresentaram associação estatisticamente significante com o peso ao nascer.

\footnotetext{
1 Mestranda em Ciências da Saúde, Faculdade de Medicina, Universidade Federal de Minas Gerais. Av. Alfredo Balena, 190, Santa Efigênia, 30130-100, Belo Horizonte, MG, Brasil. E-mail: <danisr_nutricao@yahoo.com.br>.

2 Mestranda em Ciências da Nutrição, Universidade Federal de Viçosa. Viçosa, MG, Brasil.

3 Departamento de Nutrição e Saúde, Universidade Federal de Viçosa. Av. P.H. Rolfs, s/n, Campus Universitário, 39571-000, Viçosa, MG, Brasil. Correspondência para/Correspondence to: S.C.C. FRANCESCHINI.

${ }^{4}$ Nutricionista, Prefeitura Municipal de Viçosa. Viçosa, MG, Brasil.
} 


\section{Conclusão}

As variáveis antropométricas maternas apresentaram correlação com o peso ao nascer. Neste estudo não houve relação entre o estado nutricional de ferro das gestantes e o peso de nascimento.

Termos de indexação: anemia ferropriva, estado nutricional, gestante, peso ao nascer.

\section{A B S T R A C T}

\section{Objective}

The purpose of the study was to evaluate the nutritional status and prevalence to anemia during pregnancy, and to correlate these factors with the weight at birth.

\section{Methods}

A transversal study was carried out with pregnant women who underwent prenatal care at the only public health service in the municipality of Viçosa, MG, Brazil, from December 2002 to May 2003. A questionnaire was applied to obtain information on the mothers, and their hemoglobin levels were dosed using Hemocue, also registering the mothers' height and weight. Information on the newborns was obtained from the Birth Registries Information System.

\section{Results}

A total of 168 low-income, low-schooling level pregnant women were evaluated, $41.3 \%$ showing inadequate pre-gestational nutritional status, $25.7 \%$ being underweight and $17.4 \%$ overweight or obese. The majority of the pregnant women presented inadequate weight gain during pregnancy. The prevalence of total iron deficiency anemia was $21.4 \%$, increasing with gestational age. The frequencies of low birth weight and insufficient birth weight were $8.9 \%$ and $28.6 \%$, respectively. The anthropometrical variables (pre-gestational weight, height, pre-gestational Body Mass Index and total weight gain) presented a statistically significant association with weight at birth.

\section{Conclusion}

The mother's anthropometrical variables correlated with the weight at birth. No correlation was found between the nutritional iron status of the pregnant women and weight at birth.

Indexing terms: iron-deficiency anemia, nutritional state, pregnant, birth weight.

\section{N T R O D U Ç Ã O}

O prognóstico da gestação é influenciado pelo estado nutricional materno antes e durante a gravidez. A inadequação do estado nutricional materno tem grande impacto sobre o crescimento e desenvolvimento do recém-nascido, pois o período gestacional é uma fase na qual as necessidades nutricionais são elevadas, decorrentes dos ajustes fisiológicos da gestante e das demandas de nutrientes para o crescimento fetal ${ }^{1,2}$.

O estado nutricional pré-gestacional da mulher pode interferir no processo normal da gestação. Gestantes que apresentam uma reserva inadequada de nutrientes, aliada a uma ingestão dietética insuficiente, poderão ter um comprometimento do crescimento fetal, e conseqüen- temente, do peso ao nascer. Estudos realizados em nosso meio têm demonstrado que mulheres que iniciaram a gravidez com peso inferior a $50 \mathrm{~kg}$ apresentaram maior risco de gerarem crianças com baixo peso ao nascer ${ }^{2,3}$.

A recomendação do Instituto de Medicina dos Estados Unidos (1990) para o ganho de peso gestacional em relação ao índice de massa corporal (IMC) pré-gravídico é que em gestantes que apresentam IMC $<19,8 \mathrm{~kg} / \mathrm{m}^{2}$, o ganho de peso deve variar entre $12,5-18,0 \mathrm{~kg}$, naquelas com IMC entre $19,8-26,0 \mathrm{~kg} / \mathrm{m}^{2}$, o ganho de peso gestacional deveria ficar entre $11,5-16,0 \mathrm{~kg}$, e naquelas com IMC entre 26,0-29,0kg/m² e >29,0, o ganho de peso gestacional deve ser de $7,0-11,5 \mathrm{~kg}$ e, no mínimo, $6,0 \mathrm{~kg}$, respectivamente ${ }^{4}$. Entretanto, a média de ganho de peso para mulhe- 
res em países em desenvolvimento $(5,0-9,0 \mathrm{~kg})$ está muito abaixo da recomendada e também abaixo da média de ganho de peso relatado para gestantes de países desenvolvidos $(10,5-13,5 \mathrm{~kg})^{5}$.

A relação entre o ganho de peso da gestante e o peso da criança ao nascimento é amplamente conhecida. Gestante com ganho de peso insuficiente apresenta maior risco de gerar recém-nascido com peso inadequado, podendo comprometer o crescimento pós-natal, com um maior risco de morbidade no primeiro ano de vida. Também o ganho de peso gestacional excessivo não é benéfico ao recém-nascido, pois às vezes esse excedente serve apenas para deteriorar o estado nutricional materno e, não necessariamente, é canalizado para o feto ${ }^{6,7}$. Outra variável antropométrica relacionada ao peso ao nascer é a estatura materna. Estudos verificaram que gestantes com altura inferior a $150 \mathrm{~cm}$ apresentavam maior risco de gerarem recém-nascidos de baixo peso ${ }^{8-11}$.

Segundo a World Health Organization ${ }^{12}$, a prevalência de anemia ferropriva em gestantes de países desenvolvidos e em desenvolvimento é de $22,7 \%$ e $52,0 \%$, respectivamente, sendo a prevalência total de 50,0\%. Essa elevada taxa de anemia na gestação pode estar relacionada a uma dieta insuficiente de ferro, associada ao aumento da demanda do mineral típico desse período. Uma dieta equilibrada nem sempre é suficiente para suprir as necessidades de ferro que aumentam durante a gestação, sendo necessários até dois anos para o restabelecimento dos depósitos utilizados durante esse período ${ }^{13}$. A deficiência de ferro na gestante pode acarretar efeitos adversos tanto para a sua saúde quanto para a do recém-nascido. A redução na concentração de hemoglobina na gestante resulta em aumento do débito cardíaco a fim de manter um fornecimento adequado de oxigênio via placenta às células fetais $^{14}$. As anemias maternas moderada e grave estão associadas a um aumento na incidência de abortos espontâneos, partos prematuros, baixo peso ao nascer e morte perinatal. Os efeitos no feto podem ser a restrição do crescimento intra- uterino, prematuridade, morte fetal e anemia no primeiro ano de vida, devido às baixas reservas de ferro no recém-nascido ${ }^{15,16}$.

O peso ao nascer reflete a qualidade da atenção dada à gestante durante esse período de grande vulnerabilidade, devendo haver preocupação com o seu estado nutricional, antes e durante a gestação, e observar os fatores de risco associados ${ }^{7}$. O peso ao nascer é o fator isolado mais importante na determinação da sobrevivência infantil, pois crianças com baixo peso $(\leq 2500$ gramas) apresentam maior risco de adoecer ou morrer no primeiro ano de vida. Menezes et al. ${ }^{17}$ encontraram que crianças nascidas de baixo peso apresentaram mortalidade doze vezes maior do que crianças com peso adequado ao nascer.

A taxa mundial de baixo peso ao nascer é de $14 \%$, sendo que nos países desenvolvidos e em desenvolvimento os valores são de 7\% e 15\%; respectivamente. No Brasil ${ }^{18}$, em 2003, o índice foi de $10 \%$.

Conhecendo-se a importância do estado nutricional e da anemia ferropriva durante a gestação, este estudo tem como objetivo avaliar o estado nutricional e a prevalência de anemia em gestantes e correlacioná-los com o peso da criança ao nascimento.

\section{M É T O D O S}

Realizou-se um estudo transversal no Centro de Saúde da Mulher e da Criança, Secretaria Municipal de Saúde, Prefeitura Municipal de Viçosa, Minas Gerais. Esse serviço para assistência pré-natal, com atendimento médico e nutricional, é o único para atendimento de gestantes de baixo nível socioeconômico no município.

Para o cálculo amostral, levaram-se em consideração o número de crianças nascidas em 2002 ( $n=1060)$, a freqüência de anemia em gestantes, obtida em estudo anterior (aproximadamente $15,0 \%$ ), e a variabilidade aceitável nessa freqüência de 5,0\%, totalizando tamanho amostral mínimo de 163 gestantes, com nível de confiança 
de 99,9\%. A inclusão das gestantes foi realizada no período de dezembro de 2002 a maio de 2003, totalizando 183 gestantes, das quais 15 (8,2\%) foram excluídas por falta de informação referente ao peso ao nascer no programa Sistema de Informação de Nascidos Vivos, Ministério da Saúde (SINASC).

As gestantes foram convidadas a participar do estudo e, após assinarem um termo de consentimento, foi aplicado um questionário pré-codificado por um profissional devidamente treinado contendo informações socioeconômicas, peso pré-gestacional, uso de suplementos, bem como a informação se a gestante teve algum diagnóstico de anemia durante o pré-natal.

Para avaliação antropométrica das gestantes, o peso foi verificado em balança digital eletrônica, com capacidade de $150 \mathrm{~kg}$ e divisão de $50 \mathrm{~g}$. A estatura foi aferida com fita métrica metálica, com extensão de dois metros, dividida em centímetros e subdividida em milímetros. As técnicas para obtenção dessas medidas foram as preconizadas por Jelliffe ${ }^{19}$.

Para a dosagem de hemoglobina foi realizada coleta de sangue por punção no dedo anular da mão não-dominante da gestante, sendo os níveis medidos em $\beta$-hemoglobinômetro (Hemocue), equipamento recomendado para o uso em investigações populacionais tendo em vista o pequeno volume sanguíneo necessário $(20 \mu \mathrm{L})$ e a imediata obtenção do resultado ${ }^{12}$. O ponto de corte utilizado para o diagnóstico da anemia foi de $11,0 \mathrm{~g} / \mathrm{dL}$, seguindo as recomendações do Ministério da Saúde ${ }^{20}$ bem como da Organização Mundial da Saúde ${ }^{12}$.

Os dados referentes à criança, como data de nascimento, peso ao nascer e idade gestacional, foram obtidos do programa SINASC, fornecidos pela Secretaria de Saúde. O ganho de peso total de cada gestante foi obtido do seu prontuário de acompanhamento pré-natal, no centro de saúde onde a gestante realizou o pré-natal.

O estado nutricional pré-gestacional e o ganho de peso durante a gestação foram avaliados segundo o Instituto de Medicina dos Estados Unidos ${ }^{4}$.

Os fatores de risco considerados para o baixo peso ao nascer foram: estatura inferior a $150 \mathrm{~cm}$, peso pré-gestacional inferior a $50 \mathrm{~kg}$, IMC pré-gestacional igual ou inferior a 19,8 e superior a 26,0, ganho de peso gestacional inferior a 7,0 kg e superior a $16,0 \mathrm{~kg}$. Além das variáveis antropométricas, considerou-se como risco a presença de anemia na gestação $2,6,10,11,16$. Foram consideradas como nascidas de baixo peso, crianças com peso ao nascer inferior a $2500 \mathrm{~g}$.

Os dados foram armazenados e analisados no programa computadorizado Epi Info 6.04b21. Utilizou-se ainda o programa Sigma Stat para análise estatística22.

As médias de peso ao nascer foram comparadas utilizando-se o teste " $\mathrm{t}$ " de Student no caso de dois grupos independentes e a Análise de Variância (Anova) complementada com o Procedimento de Comparações Múltiplas de Tukey, para três ou mais grupos independentes. Foi aplicado ainda o Coeficiente de Correlação de Pearson para verificar a correlação entre medidas antropométricas e hemoglobina durante a gravidez com o peso ao nascer das crianças. O nível de rejeição da hipótese de nulidade foi inferior a $0,05 \%$ ou $5,00 \%$.

Todas as gestantes receberam orientação nutricional e as diagnosticadas como anêmicas foram encaminhadas para tratamento. $O$ trabalho foi aprovado pelo Comitê de Ética em Pesquisa com Seres Humanos da Universidade Federal de Viçosa.

\section{RESULTADOS E DISCUSS Ã O}

Foram avaliadas 183 gestantes, das quais 168 foram incluídas no estudo, sendo excluídas 15 gestantes $(8,2 \%)$ por falta de informações referentes ao peso ao nascer no SINASC. Destaca-se que essa perda amostral pode ter ocorrido em função de aborto, mudanças de município ou parto domiciliar, resultando em sub-registro de 
nascidos no SINASC, em relação aos atendimentos realizados no Centro de Saúde da Mulher e da Criança. Isso poderia ser evitado se houvesse maior eficiência na coleta de informações que mantêm a base de dados dos indicadores de saúde do município.

Em relação à idade gestacional, 10,7\% estavam no primeiro trimestre de gestação, $44,0 \%$ no segundo e $45,3 \%$ no último trimestre de gestação. Das gestantes avaliadas, 27,4\% eram adolescentes, 3,0\% possuíam mais de 35 anos e a maioria, 69,6\%, tinha entre 20 e 35 anos de idade. A idade apresentou variabilidade entre 14 e 38 anos, sendo a média de $23,6 \pm 5,4$ anos.

A maior parte das gestantes $(70,9 \%)$ iniciou o pré-natal no primeiro trimestre de gestação e apenas quatro $(2,4 \%)$ no último trimestre. Com relação à paridade, $41,7 \%$ das gestantes estavam na primeira gestação e 58,3\% eram multíparas, sendo que, dentre essas, 10,3\% eram grandes multíparas (quatro filhos ou mais).

Em relação à escolaridade, a grande maioria das gestantes $(61,3 \%)$ não tinha o primeiro grau completo e apenas $2,4 \%$ tinham doze ou mais anos de estudo. A média de escolaridade das gestantes avaliadas foi de $6,7 \pm 3,1$ anos. Com relação à renda familiar, $77,6 \%$ das gestantes disseram receber três salários mínimos ou menos, e, dentre essas, 12,3\% pertenciam a famílias que recebiam menos de um salário-mínimo. A partir desses dados, observa-se que a população estudada caracteriza-se por gestantes de baixo nível socioeconômico e baixa escolaridade.

O cuidado nutricional pré-natal tem demonstrado impacto positivo sobre os resultados obstétricos. A identificação precoce de gestantes com inadequação no estado nutricional permite uma melhora do estado nutricional materno, tem impacto positivo nas condições ao nascer, podendo minimizar as taxas de morbimortalidade perinatal e neonatal ${ }^{1,2}$. O peso pré-gestacional é um determinante significativo do peso da criança ao nascer. No presente estudo, a freqüência de mulheres que iniciaram a gravidez com peso inferior a $50 \mathrm{~kg}$ foi de $25,7 \%$, freqüência superior à encontrada em estudo realizado em Pelotas, no qual a freqüência de gestantes com peso pré-gestacional inferior a $50 \mathrm{~kg}$ foi de $15,7 \%{ }^{23}$.

Outra variável relacionada ao peso ao nascer é a estatura materna. Mulheres com altura inferior a 1,50m apresentam maior probabilidade de gerarem filhos de baixo peso ao nascer, além do risco da desproporção céfalo-pélvica ${ }^{1,11}$. Neste estudo, 7,1\% das gestantes avaliadas tinham altura inferior a 1,50m, com média de $1,59 \pm 0,07 \mathrm{~m}$. Pode-se observar (Tabela 1) que $25,7 \%$ das gestantes iniciaram a gravidez com baixo peso. Esse dado é relevante, pois gestantes com reserva inadequada de energia podem comprometer o crescimento fetal e, conseqüentemente, o peso da criança ao nascer ${ }^{24}$. Por outro lado, deve-se preocupar também com mulheres que iniciam a gravidez com excesso de peso. O ganho de peso materno excessivo pode levar a complicações gestacionais, como diabetes, macrossomia e distúrbios hipertensivos ${ }^{24}$. Nucci et al. ${ }^{24}$ encontraram prevalências de $5,7 \%$ de baixo peso, $19,2 \%$ de sobrepeso e $5,5 \%$ de obesidade, considerando-se o peso pré-gestacional de gestantes em seis capitais brasileiras entre 1991 e 1995. Os dados de baixo peso e obesidade nas gestantes do município de Viçosa, MG indicam elevadas taxas de prevalência.

Outra variável a ser considerada em conjunto com o peso pré-gestacional é o ganho de peso durante a gestação. Pode-se observar

Tabela 1. Estado nutricional pré-gravídico das gestantes avaliadas no Centro de Saúde da Mulher e da Criança, município de Viçosa, MG.

\begin{tabular}{lcr}
\hline Estado nutricional & $\mathrm{n}$ & $\%$ \\
\hline Baixo peso & 43 & 25,7 \\
Eutrófica & 95 & 56,9 \\
Sobrepeso & 13 & 7,8 \\
Obesidade & 16 & 9,6 \\
\hline Total & $167^{*}$ & 100,0 \\
\hline
\end{tabular}

* Uma gestante não soube informar seu peso pré-gestacional. 
(Tabela 2) que, entre o grupo de gestantes de baixo peso, quase $60,0 \%$ apresentaram ganho de peso insuficiente até o momento da avaliação, tendo essas gestantes maior risco de gerarem crianças nascidas com baixo peso. Nesse mesmo grupo, 18,6\% apresentaram ganho excessivo de peso. Entre o grupo de gestantes eutróficas, $51,6 \%$ apresentaram reduzido ganho de peso e $26,3 \%$ elevado ganho de peso gestacional. No grupo das gestantes com sobrepeso e obesidade, a maioria $(48,3 \%)$ teve um ganho de peso excessivo. Esses dados demonstram a importância de uma orientação nutricional individualizada com o objetivo de melhorar o estado nutricional materno, reduzindo os riscos de intercorrências durante a gestação, no parto e o de baixo peso ao nascer.

Com relação ao ganho de peso total durante a gestação, 19,9\% das gestantes ganharam menos de $7,0 \mathrm{~kg} \mathrm{e} 12,1 \%$ mais de $16 \mathrm{~kg}$. A média do ganho de peso gestacional foi de $11,0 \pm 4,4 \mathrm{~kg}$.

Considera-se a gestante vulnerável à anemia ferropriva pelo aumento das necessidades desse mineral devido não só ao crescimento fetal e placentário, como também ao aumento da volemia, decorrente da gravidez ${ }^{25}$. Neste estudo, $17,3 \%$ das gestantes relataram ter tido anemia no início da gestação, momento em que deve ser feito o hemograma, considerado exame obrigatório. Durante a realização do teste de anemia por meio do Hemocue, foi encontrada prevalência superior $(21,4 \%)$, com média de hemoglobina de $11,7 \pm 1,2 \mathrm{~g} / \mathrm{dL}$. A prevalência de anemia aumentou com a idade gestacional, sendo $5,6 \%, 20,3 \%$ e $26,3 \%$ para o primeiro, segundo e terceiro trimestre de gestação, respectivamente. Com relação às médias de hemoglobina, encontrou-se $12,5 \pm 1,1 \mathrm{~g} / \mathrm{dL}, 11,6 \pm 1,1 \mathrm{~g} / \mathrm{dL}$ e $11,6 \pm 1,2 \mathrm{~g} / \mathrm{dL}$, no primeiro, segundo e terceiro trimestre de gestação, respectivamente. Esses dados indicam um aumento na prevalência de anemia entre gestantes atendidas nesse Centro de Saúde, pois, em 1998, Rosado ${ }^{26}$ encontrou prevalência de $14,5 \%$ de anemia em gestantes adolescentes atendidas no mesmo serviço de saúde.

Em relação à ingestão de composto ferroso pelas gestantes, observou-se que 35,7\% $(n=60)$ não tinham tomado ainda sulfato ferroso ou suplemento vitamínico, e, entre essas, 21,6\% estavam com vinte ou mais semanas de gestação. Segundo o Ministério da Saúde ${ }^{20}$, a profilaxia deve ser feita a partir da vigésima semana, utilizando-se uma drágea de sulfato ferroso ao dia (300mg), que corresponde a $60 \mathrm{mg}$ de ferro elementar, que deve ser ingerido 30 minutos antes das refeições. Foi relatado por $79,0 \%$ das gestantes que a ingestão era feita junto com as grandes refeições, o que pode levar a uma interferência na absorção de ferro medicamentoso.

Encontraram-se $8,9 \%$ de baixo peso ao nascer (<2 500g) na população estudada, 28,6\% de peso insuficiente (2 500-2 999g) e 62,5\% de peso adequado $(\geq 3000 \mathrm{~g})$, com média de $3166 \pm 528 \mathrm{~g}$. A porcentagem de baixo peso ao nascer na amostra estudada foi inferior à incidência do ano de 2003 no Brasil (10,0\%)

Tabela 2. Adequação do ganho de peso em função do estado nutricional pré-gravídico das gestantes estudadas no Centro de Saúde da Mulher e da Criança, município de Viçosa, MG.

\begin{tabular}{|c|c|c|c|c|c|c|c|c|}
\hline \multirow{3}{*}{ Ganho de Peso } & \multicolumn{6}{|c|}{ Estado nutricional pré-gestacional } & & \\
\hline & \multicolumn{2}{|c|}{ Baixo peso } & \multicolumn{2}{|c|}{ Eutróficas } & \multicolumn{2}{|c|}{ Sobrepeso/obesidade } & \multicolumn{2}{|c|}{ Total } \\
\hline & $\mathrm{n}$ & $\%$ & $\mathrm{n}$ & $\%$ & $\mathrm{n}$ & $\%$ & $\mathrm{n}$ & $\%$ \\
\hline Insuficiente & 25 & 58,1 & 49 & 51,6 & 10 & 34,5 & 84 & 50,3 \\
\hline Adequado & 10 & 23,3 & 21 & 22,1 & 5 & 17,2 & 36 & 21,6 \\
\hline Excessivo & 8 & 18,6 & 25 & 26,3 & 14 & 48,3 & 47 & 28,1 \\
\hline Total & 43 & 100,0 & 95 & 100,0 & 29 & 100,0 & 167 & 100,0 \\
\hline
\end{tabular}

* Uma gestante não sabia informar o peso pré-gestacional. 
Porém, o índice observado pode ser considerado elevado, tendo em vista o seu impacto sobre a saúde infantil. Recém-nascidos de baixo peso apresentam probabilidade quarenta vezes maior de morrerem no período neonatal quando comparados com os de peso adequado ${ }^{27}$.

Com relação ao estado nutricional materno, anemia e peso ao nascer, são apresentadas algumas características do estado nutricional e presença de anemia das gestantes avaliadas, a freqüência dessas características presentes na população e sua relação com o peso ao nascer (Tabela 3).

Observa-se, a partir da Tabela 3, que as variáveis antropométricas (peso pré-gestacional, altura, IMC pré-gestacional e ganho de peso total durante a gestação) apresentaram relação estatisticamente significante com o peso ao nascer, dado não encontrado em relação à presença de anemia durante a gestação. Em relação ao IMC pré-gestacional, a diferença significante entre as médias de peso ao nascer ocorreu entre as gestantes que iniciaram a gestação de baixo peso em relação àquelas com sobrepeso ou obesidade.
Já para o ganho de peso total, a diferença foi entre gestantes com ganho de peso insuficiente em relação àquelas com ganho de peso adequado e/ou excessivo.

Verifica-se, na Tabela 4, que todas as variáveis antropométricas maternas apresentaram correlação fraca, porém estatisticamente significante com o peso ao nascer. Dentre essas variáveis, o ganho de peso total apresentou maior correlação. Como já evidenciado também na comparação entre as médias (Tabela 3), a hemoglobina materna não apresentou correlação com o peso ao nascer.

Tabela 4. Coeficiente de Correlação entre peso ao nascer com medidas antropométricas e hemoglobina materna, município de Viçosa, MG.

\begin{tabular}{lcc}
\hline Características gestacionais & \multicolumn{2}{c}{ Peso ao nascer } \\
\cline { 2 - 3 } maternas & $r$ & $p$ \\
\hline Peso pré-gestacional & 0,27 & $<0,0005$ \\
IMC pré-gestacional & 0,16 & $<0,05$ \\
Altura & 0,23 & $<0,005$ \\
Ganho de peso total & 0,37 & $<0,0001$ \\
Hemoglobina & 0,04 & 0,63 \\
\hline
\end{tabular}

$r=$ coeficiente de correlação.

Tabela 3. Peso ao nascer segundo características gestacionais, município de Viçosa, MG.

\begin{tabular}{|c|c|c|c|c|c|c|}
\hline \multirow{2}{*}{ Características gestacionais } & \multicolumn{2}{|c|}{ Freqüência } & \multicolumn{2}{|c|}{ Peso ao nascer } & \multirow{2}{*}{ Estatística calculadaa } & \multirow{2}{*}{$p$} \\
\hline & $n$ & $\%$ & M & $\pm \mathrm{DP}$ & & \\
\hline \multicolumn{7}{|l|}{ Peso pré-gestacional } \\
\hline$<50 \mathrm{~kg}$ & 43 & 25,7 & 2976 & \pm 459 & $t=2,85$ & $<0,005$ \\
\hline$\geq 50 \mathrm{~kg}$ & 124 & 74,3 & 3236 & \pm 535 & & \\
\hline \multicolumn{7}{|l|}{ Estatura } \\
\hline$<150 \mathrm{~cm}$ & 12 & 7,1 & 2702 & \pm 404 & $t=3,25$ & $<0,001$ \\
\hline$\geq 150 \mathrm{~cm}$ & 156 & 92,9 & 3201 & \pm 521 & & \\
\hline \multicolumn{7}{|l|}{ IMC pré-gestacional $\left(\mathrm{kg} / \mathrm{m}^{2}\right)^{\mathrm{b}}$} \\
\hline Baixo Peso $(<19,8)$ & 43 & 25,7 & 3076 & $\pm 483^{*}$ & $F=2,89$ & 0,05 \\
\hline Eutrófica $(19,8|-| 26)$ & 95 & 56,9 & 3151 & \pm 512 & & \\
\hline Sobrepeso/Obesidade (>26) & 29 & 17,4 & 3369 & \pm 606 & & \\
\hline \multicolumn{7}{|l|}{ Ganho de peso total $(\mathrm{kg})^{c}$} \\
\hline Insuficiente $(<7,0)$ & 28 & 19,9 & 2856 & $\pm 438^{* *}$ & $F=10,70$ & $<0,005$ \\
\hline Adequada $(7,0|-| 16,0)$ & 96 & 68,1 & 3173 & \pm 505 & & \\
\hline Excessivo $(>16,0)$ & 17 & 12,0 & 3566 & \pm 572 & & \\
\hline \multicolumn{7}{|l|}{ Presença de Anemia } \\
\hline Sim & 36 & 21,4 & 3103 & \pm 453 & $t=0,80$ & 0,400 \\
\hline Não & 132 & 78,6 & 3183 & \pm 547 & & \\
\hline
\end{tabular}

${ }^{a} \mathrm{t}=$ Teste " $\mathrm{t}$ " de Student; $\mathrm{F}=$ Análise de variância; ${ }^{\mathrm{b}} \mathrm{n}=167$ (uma gestante não sabia informar o peso pré-gestacional); ${ }^{\mathrm{c}} \mathrm{n}=141$ (27 prontuários não possuíam a evolução do peso da gestante); * Baixo peso<sobrepeso/obesidade; **Insuficiente<Adequação; Sobrepeso. 
A avaliação do estado nutricional das gestantes de baixo nível socioeconômico identificou uma alta prevalência de gestantes com estado nutricional desfavorável, pois encontraram-se, aproximadamente, $43,0 \%$ de gestantes que iniciaram o pré-natal com baixo peso, sobrepeso e obesidade. Aliado a isso, observou-se que, entre as gestantes de baixo peso, quase $60,0 \%$ apresentaram ganho de peso insuficiente. Ainda, 21,4\% encontravam-se anêmicas, sendo que a anemia aumentou com a idade gestacional, atingindo $26,3 \%$ no último trimestre de gestação. A anemia na gravidez pode ser devida à qualidade da assistência pré-natal relacionada à prevenção e tratamento da anemia ferropriva, uma vez que $35,0 \%$ das gestantes não estavam recebendo suplemento.

Ao correlacionar as variáveis antropométricas das gestantes com o peso ao nascer, observou-se que todas as variáveis antropométricas apresentaram relação estatisticamente significante com o peso ao nascer, sendo que o ganho de peso gestacional apresentou maior correlação $(r=0,37)$. Nesse estudo não houve relação entre o estado nutricional de ferro das gestantes com o peso de nascimento.

Informações deste estudo são importantes para orientar as medidas necessárias que visam reduzir os fatores de risco para o baixo peso ao nascer e a prevalência de anemia nessa população. A atenção nutricional à gestante bem como a profilaxia com ferro devem integrar a assistência pré-natal, cuja qualidade se baseia no início precoce, número mínimo de consultas, identificação e maior atenção às gestantes de alto risco, combate ao tabagismo durante a gestação, dentre outras ações com impacto conhecido sobre o peso ao nascer.

\section{A GRADECIMENTOS}

À nutricionista do Centro de Saúde Cláudia Antunes, pela ajuda na inclusão das gestantes. Ao apoio financeiro do CNPq e da Prefeitura Municipal de Viçosa.
1. Accioly E, Saunders C, Lacerda EMA. Nutrição em obstetrícia e pediatria. Rio de Janeiro: Cultura Médica; 2002. 540p.

2. Ramakrishman U. Nutrition and low birth weight: from research to practice. Am J Clin Nutr. 2004; 79(1):17-21.

3. Halpern R, Schaefer ES, Pereira AS, Arnt EM, Bezerra JPV, Pinto LS. Fatores de risco para o baixo peso ao nascer em uma comunidade rural do sul do Brasil. J Pediatr. 1996; 72(6):369-73.

4. Institute of Medicine. Nutrition during pregnancy, weight gain and nutrient supplements. Report of the Subcommitee on Nutritional Status and weight gain during Pregnancy, Subcommitee on Dietary Intake and Nutrient Supplements during Pregnancy, Committee on Nutritional Status during Pregnancy and Lactation, Food and Nutrition Board. Washington (DC): National Academy Press; 1990.

5. Krasovec K, Anderson MA. Maternal Nutrition and pregnancy outcomes. Anthropometric Assessment. Washington (DC): Scientific Publication; 1991.

6. Lizo CLP, Azevedo-Lizo Z, Aronson E, Segre CAM. Relação entre ganho de peso materno e peso do recém-nascido. J Pediatr. 1998; 74(2):114-8.

7. Abrams B, Altman SL, Pickett KE. Pregnancy weight gain: still controversial. Am J Clin Nutr. 2000; 71(Suppl):S1233-41.

8. Siqueira $A A F$, et al. Influência da altura e ganho de peso materno e da idade gestacional sobre o peso do recém-nascido: estudo de 3 grupos de gestantes normais. Rev Saúde Pública. 1975; 9:331-42.

9. Ciari CJ, Almeida PAM, Siqueira AAF. Relação entre peso da criança ao nascer, altura materna, idade gestacional e restrição alimentar em gestantes normais. Rev Saúde Pública. 1975; 9:33-42.

10. Franceschini SCC, Priore SE, Pequeno NPF, Silva DG, Sigulem DM. Fatores de risco para o baixo peso ao nascer em gestantes de baixa renda. Rev Nutr. 2003; 16(2):171-9.

11. Zambonato AMK, Pinheiro RT, Horta BE, Tomasi E. Fatores de risco para nascimento de crianças pequenas para idade gestacional. Rev Saúde Pública. 2004; 38(1):24-9.

12. World Health Organization. Iron deficiency anaemia: assessment, prevention, and control: a guide for programme managers. Geneva: WHO; 2001.

13. Guerra EM, Barretto OCO, Pinto AV, Castellão KG. Prevalência de deficiência de ferro em gestantes 
de primeira consulta em centros de saúde de área metropolitana, Brasil. Etiologia da anemia. Rev Saúde Pública. 1992; 26(2):88-95.

14. Worthington-Roberts B, Williams SR. Nutrition in pregnancy and lactation. United States: Copyright; 1993.

15. Laborda RGA, González EF. Nutrición y dietética durante el embarazo. Barcelona: Talleres Gráficos Dúplex; 1996.

16. Rasmussen KM. Is there a causal relationship between iron deficiency or iron-deficiency anemia and weight at birth, length of gestation and perinatal mortality? J Nutr. 2001:590s-603.

17. Menezes AMB, Victora CG, Barros FC. Mortalidade infantil em duas coortes de base populacional no Sul do Brasil: tendências e diferenciais. Cad Saúde Pública. 1996; 12(1):79-86.

18. United Nations Children's Fund. The state of the world's children, 2004. New York: UNICEF; 2004.

19. Jelliffe $D B$. The assessment of the nutritional status of the community. Geneva; WHO; 1968.

20. Ministério da Saúde. Assistência pré-natal. Manual técnico. Brasília; 2000.

21. Dean AG, Dean JA, Burton AH, Dicker RC. Epi Info 6.04: a word processing, database, and statistics program for epidemiology on micro-computers. Georgia: Center for Disease Control; 1990.

22. Fox $E$, Kuo J, Tilling L, Ulrich C. User's manual sigma stat: statistical software for windows. Berlim: Jandel; 1994.

23. Halpern R, Barros FC, Victora CG, Tomasi E. Atenção pré-natal em Pelotas, Rio Grande do Sul, Brasil, 1993. Cad Saúde Pública. 1998; 14(3):487-92.

24. Nucci LB, Schmidt MI, Duncan BB, Fuchs SC, Fleck ET, Britto MMS. Nutritional status of pregnant women: prevalence and associated pregnancy outcomes. Rev Saúde Pública. 2001; 35(6):502-7.

25. Martins IS, Alvarenga AT, Siqueira AAF, Szarfarc SC, Lima FD. As determinações biológica e social da doença: um estudo de anemia ferropriva. Rev Saúde Pública. 1987; 21(2):73-89.

26. Rosado LEFPL. Estado nutricional de gestantes adolescentes e sua relação com o peso do recémnascido em Viçosa, MG [tese]. São Paulo: Universidade Federal de São Paulo; 1998.

27. Euclydes MP. Nutrição do lactente: base científica para uma alimentação adequada. Viçosa: Universidade Federal de Viçosa; 2000.

Recebido para publicação em 21 de junho de 2004 e aceito em 16 de fevereiro de 2005. 